\title{
Characterization of Protein N-Glycosylation by Reversed-Phase Microbore Liquid Chromatography / Electrospray Mass Spectrometry, Complementary Mobile Phases, and Sequential Exoglycosidase Digestion
}

\author{
K. F. Medzihradszky, D. A. Maltby*, S. C. Hall, C. A. Settineri, and \\ A. L. Burlingame* \\ Department of Pharmaceutical Chemistry, Mass Spectrometry Facility, and "the Liver Center, University of \\ California, San Francisco, Califorria, USA
}

\begin{abstract}
A strategy for the identification of the site occupancy and glycoform heterogeneity, including sialylation occurring at specific sites of $N$-linked glycoproteins is presented using the asparagine-linked glycosylation on bovine fetuin for illustration. This is achieved by microbore high-performance liquid chromatography/electrospray ionization mass analysis (LC/ESIMS) of the tryptic glycopeptide mixtures with an acetonitrile-based mobile phase followed by sequential steps of residue (and linkage) specific glycoform degradation and LC/ESIMS analysis at each stage. In addition, chromatographic separation of the site-specific glycoforms of tryptic glycopeptides is accomplished by the use of an alternative, mass spectrometrically compatible mobile phase - water/ethanol/propanol/formic acid. By employing this nontraditional mobile phase for characterizing the complete tryptic digest, and using highly specific exoglycosidases in combination with LC/ESIMS analysis, a previously uncharacterized carbohydrate (a disialo biantennary complex oligosaccharide) was identified as a novel structure at $\mathrm{Asn}^{81}$ of bovine fetuin. (J Am Soc Mass Spectrom 1994, 5, 350-358)
\end{abstract}

$\mathrm{T}$ The characterization of the gross covalent structure of glycoproteins has been revolutionized with the advent of capillary and microbore highperformance liquid chromatography (HPLC) combined with electrospray ionization mass spectrometry (LC/ESIMS). Using a combination of proteolytic enzymatic digestion and separation of the digest mixture by HPLC coupled directly with electrospray mass spectrometry, a glycoprotein mass map can be obtained consisting of both peptides and $N$ - and $O$-linked glycopeptides [1-4], and the nature and extent of site heterogeneity, including sialylation, of intact native glycopeptides may also be investigated [3-5]. The location of those components bearing glycosylation in such digests is usually difficult because of inadequate chromatographic resolution $[1,6]$. However, as recently reported by Carr et al., collisional activation of electrospray generated ions (either in the source or within the second quadrupole of a triple quadrupole mass analyzer) induces dissociation reactions preferentially within the carbohydrate moieties themselves, forming fragment ions specific to the oligosaccharide

Address reprint requests to Professor A. L. Burlingame, Department of Pharmaceutical Chemistry, University of California, San Francisco, San Francisco, CA 94143-U446. structures attached to the peptide backbone $[3,5,7]$. Hence, monitoring of these particular lower mass carbohydrate ions throughout the chromatogram permits detection of glycosylated species and of course, measurement of their retention times. However, because of the complexity of carbohydrate heterogeneity which is often present within and among sites in a glycoprotein, it is not possible in most cases to completely resolve the individual glycoforms using only one chromatographic separation method. Depending on the degree of glycosylation, several glycoforms of the same amino acid sequence usually coelute, making interpretation of such mass spectra difficult. In addition, when several components (whether peptides and glycopeptides or glycopeptide glycoforms) coelute, possible problems with suppression and/or usable dynamic range may arise, especially when attempting to detect and measure nonstoichiometric quantities of glycopeptides by LC/ESIMS. Reversed-phase separation of $N$-linked glycopeptides is mainly influenced by the hydrophilicity /hydrophobicity ratio of the particular peptide sequence, the carbohydrate structure having only minimal effect. In the commonly used water/acetonitrile/ TFA (TFA = trifluoroacetic acid) solvent system species of the same amino acid sequence with the largest carbohydrate structure (most hydrophilic) elute 
first, as one would expect, followed by the less glycosylated components. Improved chromatographic resolution can be achieved by decreasing the flow rate, reducing the stationary phase particle diameter, or using a shallower gradient. However, variation of these parameters will not yield substantial changes in the separation of such components. An alternative, more effective strategy would be selection of a different mobile phase.

Recently, interest has been expressed in using ethanol and propanol as the organic modifier during peptide and protein HPLC separations in lieu of acetonitrile $[8,9]$. Originally this choice was based strictly on concern over unknown ramifications to human health of chronic low-level exposure to acetonitrile [8]. In addition to being nontoxic, it has been reported that using an aqueous ethanol/propanol/formic acid mobile phase not only provides chromatographic resolution comparable to acetonitrile-based mobile phases, but also improves detection sensitivity for ESIMS relative to the commonly used acetonitrile mixtures [9]. The effect of using this type of solvent system on the reversed-phase chromatographic separation of glycopeptide glycoforms has not previously been investigated.

In this article we report the use of an ethanol/pro$\mathrm{panol} /$ water/formic acid solvent system for the reversed-phase separation and electrospray mass spectrometric detection of tryptic peptides and glycopeptides from bovine fetuin. Our studies presented here focus on a comparison of the performance of this mobile phase with the established acetonitrile-based solvent systems on separation of bovine fetuin $\mathrm{N}$ linked glycopeptides. Usually knowing just the values of the molecular mass(es) determined for any given glycopeptide permits postulation of the class and branching of common known oligosaccharides which may be present, but clearly does not provide prima facie evidence of structure. Thus, it is important to obtain additional experimental evidence to establish actual corresponding structure(s). For this purpose we have explored the utility of using sequential, specific exoglycosidase digestions with concomitant LC/ESIMS mass measurements on residue and linkage specific truncated glycopeptide mixtures. The results and effectiveness of this strategy in establishing carbohydrate structures present on individual glycosylation sites are described.

\section{Experimental}

\section{Materials}

Bovine fetuin was obtained from Gibco. TPCK( $\mathrm{N}^{\alpha}{ }_{-}$ tosyl-L-phenylalonyl chloromethane)-trypsin, $\mathrm{N}$-ethylmorpholine, acetic acid, and spectroscopic grade TFA were from Pierce. Vinylpyridine and formic acid were from Sigma. HPLC grade water, acetonitrile, propanol, and ethanol were from Fisher Scientific.
Neuraminidase Arthrobacter ureafaciens was from Boehringer Mannheim, and $\beta$-galactosidase from Streptococcus pneumoniae was obtained from Oxford Glycosystems.

\section{Tryptic Digestion}

Bovine fetuin (100 mg) was dissolved in a $6 \mathrm{M}$ guanidine $\mathrm{HCl} / 300 \mathrm{mM}$ N-ethylmorpholine acetate solution ( $\mathrm{pH} \mathrm{8.3)} \mathrm{and} \mathrm{was} \mathrm{reduced} \mathrm{with} \mathrm{DTT}(24 \mathrm{mg}, 4 \mathrm{~h}$, room temperature) and alkylated with vinylpyridine ( $48 \mu \mathrm{L}$, room temperature, overnight). The reduced and alkylated bovine fetuin was dialyzed in $100 \mathrm{mM}$ ammonium bicarbonate with $0.1 \mathrm{mM} \mathrm{CaCl}, \mathrm{pH} 7.8$. TPCKtrypsin, $1 \%(\mathrm{w} / \mathrm{w})$, was added to the solution and the mixture was incubated at $37^{\circ} \mathrm{C}$ for $1 \mathrm{~h}$ followed by addition of another trypsin aliquot. After $1.5 \mathrm{~h}$ the digestion was stopped by boiling. Prior to analysis, an aliquot of the digest was diluted with solvent $A$ (see below) to a final concentration of 4 picomoles $/ \mu \mathrm{L}$ from which aliquots were injected onto the HPLC column. Protein concentration data were obtained by amino acid analysis.

\section{Exoglycosidase Digestions}

Sequential exoglycosidase digestions were started with approximately 1 nmole of the tryptic glycopeptides. Neuraminidase digestions were carried out in a $30 \mathrm{mM}$ sodium acetate buffer ( $\mathrm{pH} 5.0)$ at $37{ }^{\circ} \mathrm{C}$, for $18-24 \mathrm{~h}$. Aliquots of 20 mUnits $(2 \mu \mathrm{L})$ were added at 0 and $8 \mathrm{~h}$. $\beta$-Galactosidase digestions were performed in a $30 \mathrm{mM}$ sodium acetate buffer $(\mathrm{pH} 6.0)$ at $37^{\circ} \mathrm{C}$ for $18-24 \mathrm{~h}$. Aliquots of the enzyme ( $0.8 \mathrm{mUnits})$ were added to the digestion mixture at 0 and $8 \mathrm{~h}$.

\section{Instrumentation}

A dual syringe pump (Carlo Erba or Applied Biosystems) was used to deliver mobile phase at $50 \mu \mathrm{L} / \mathrm{min}$. Microbore HPLC separations were performed on an Aquapore $300 \mathrm{C} 18$ microbore column, $1.0 \mathrm{~mm}$ i.d. $\times 100$ mm (Applied Biosystems). Twenty picomole-aliquots of the fetuin tryptic digest were injected (Rheodyne, model 8125) onto the column. Column effluent was monitored by a variable wavelength UV detector (Applied Biosystems) equipped with a high sensitivity capillary flow cell (LC Packings) at $215 \mathrm{~mm}$. Separations performed with $0.1 \%$ TFA in water as solvent $A$ and $0.08 \%$ TFA in acetonitrile as solvent $B$ required postculumn addition of 2-methoxyethanol/isopropanol (1:1) [10]. This was needed to create an eluant more conducive to electrospray ionization during the early part of the reversed phase gradient so that the hydrophilic peptide and glycopeptide mass spectrometric detection would not be compromised. A separate syringe pump (Isco) connected to a $3.1 \mu \mathrm{L}$ dead volume PEEK (polyether ether ketane) mixing tee (Upchurch Scientific), positioned after the UV detector was used to accomplish this. After the mixing tee, a zero 
dead volume tee was incorporated to split the column. effluent 1:20 so that the flow rate into the mass spectrometer was 3-5 $\mu \mathrm{L} / \mathrm{min}$, while the remaining sample was manually collected for subsequent analyses. Splitting was accomplished by using an appropriate length of fused silica capillary tubing. When solvent $A$ was $0.1 \%$ formic acid in water and solvent $B$ was $0.08 \%$ formic acid in ethanol/propanol (5:2), the addition of a make-up solvent was not necessary, and optimal sensitivity was achieved without splitting the flow. The gradient for both solvent systems was started immediately after the sample injection from $2 \% \mathrm{~B}$, and solvent $B$ was linearly increased to $50 \%$ over $50 \mathrm{~min}$.

The microbore HPLC system was interfaced to either a VG Biotech/Fisons Bio-Q or Platform mass spectrometer equipped with an electrospray source by an approximately 1 meter length of fused silica capillary tubing. The selected ion monitoring (SIM) experiments and some full scan LC/ESIMS experiments employing the acetonitrile-based solvent system were carried out on the Bio-Q mass spectrometer (Figures 1-5), while the comparison of the two solvent systems was performed using the Platform electrospray instrument. Typical operating voltages were as follows: probe tip $4200 \mathrm{~V}$, counter electrode $550 \mathrm{~V}$, and sampling orifice $40-50 \mathrm{~V}$. The source temperature was maintained at 60 ${ }^{\circ} \mathrm{C}$. The mass spectrometer was scanned in a noncontinuum mode over a range of $m / z 350-2200$ at 5 sec/scan.

In the STM experiments masses at $m / z 204,274$, 292, and 366 were monitored $(m / z$ span 0.5 ; time span $0.23 \mathrm{sec}$; interscan time $0.02 \mathrm{sec}$ ). Fragmentation

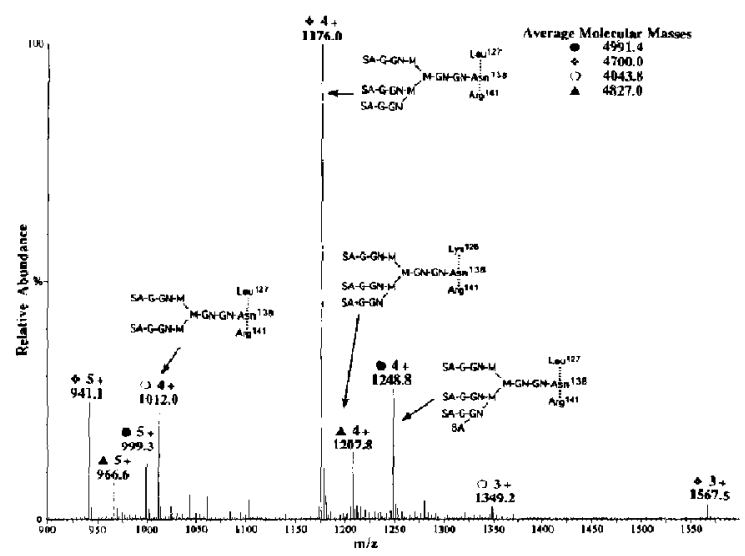

Figure 1. Electrospray mass spectrum of the bovine fetuin tryptic glycopeptides containing $\mathrm{Asn}^{138}$. This spectrum was recorded in an LC/ESIMS experiment from 20 pmoles of the tryptic digest, using water/acetonitrile/TFA as mobile phase for the chromatographic separation. Three different complex carbohydrates are linked to $\mathrm{AsI}^{138}$ : disialo biantennary, tri-, and tetrasialo triantennary structures. The calculated molecular masses for these tryptic species (amino acids 127-141) are 4043.2, 4699.8, and 4991.1 Da, respectively. The fourth glycopeptide component of this mixture is peptide 126-141 bearing a trisialo triantennary oligosaccharide $(\mathrm{MW}=4828.0 \mathrm{Da}) .(\mathrm{GN}=\mathrm{N}$-Acetyl-glucosamine; $\mathrm{M}=$ mannose; $\mathrm{G}=$ galactose; $\mathrm{S} \Lambda$ = sialic acid.)

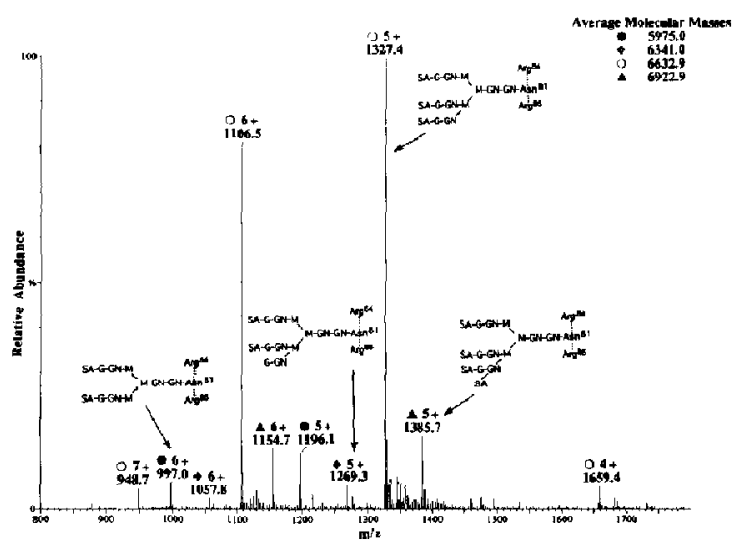

Figure 2. Electrospray mass spectrum of the bovine fetuin tryptic glycopeptides containing Asn ${ }^{81}$. This spectrum was recorded in an LC/ESIMS experiment from 20 pmoles of the tryptic digest, using water/acetonitrile/TFA as mobile phase for the chromatographic separation. Four different complex carbohydrates are linked to $\mathrm{Asn}^{81}$; disialo biantenrary, di-, tri-, and tetrasialo trianternary structures. The calculated molecular masses for these species are 5975.3,6340.5,6631.9, and 6923.3 Da, respectively. (GN - N-Acetyl-glucosamine; $M$ - malunose; $G$ - galaclose; SA = sialic acid.)

was induced in the electrospray ionization source of the Bio-Q mass spectrometer by increasing the potential of the sample cone lens to $140-200 \mathrm{~V}$ without changing any other parameters.

\section{Results}

Bovine fetuin has been studied extensively and is known to contain three $\mathrm{N}$-linked glycasylation sites

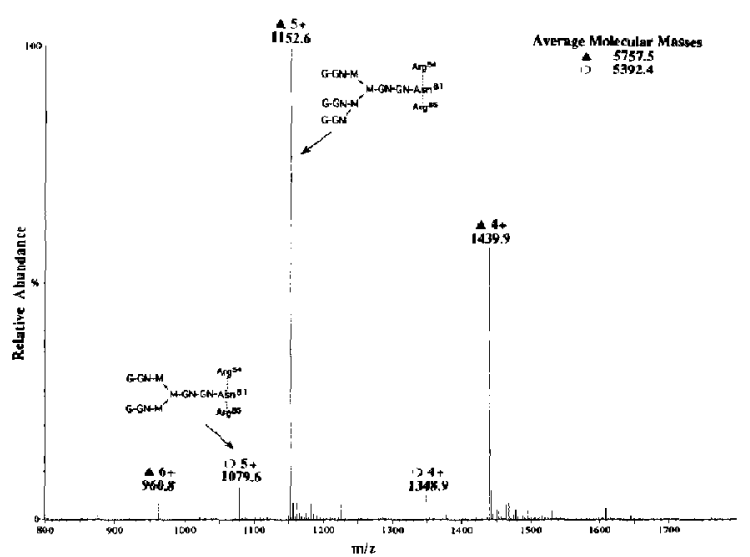

Figure 3. Electrospray mass spectrum of the neuraminidase treated fetuin tryptic glycopeptides containing Asn ${ }^{81}$. The spectrum was recorded in an LC/ESIMS experiment, using water/ acetonitrile/TFA as mobile phase for the chromatographic separation. After removing the sialic acids, glycopeptides with complex bi- and triantennary structures were observed. Calculated molecular masses for these components are 5392.8 and $5758.1 \mathrm{Da}$, respectively. $(\mathrm{CN}=\mathrm{N}$-Acetyl-glucosamine; $\mathbf{M}=-$ mannose; $\mathbf{G}=$ galactose.) 


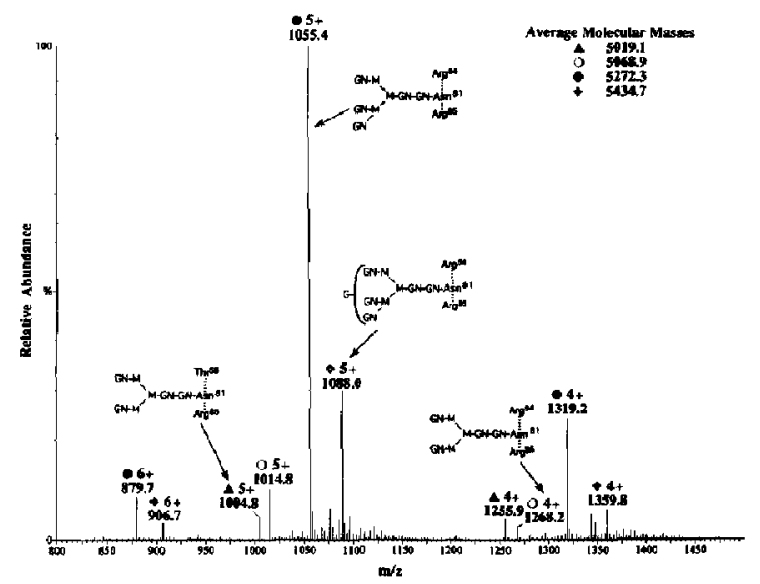

Figure 4. Electrospray mass spectrum of the $\beta$-galactosidase treated asialo-glycopeptides shown in Figure 3. This spectrum was recorded in an LC/ESIMS experiment, using water/ acetonitrile / TFA as the mobile phase for the chromatographic separation. The calculated molecular mass for glycopeptide $54-85$ with a triantennary structure, and all galactose residues removed is $5271.9 \mathrm{Da}$. The same amino acid sequence with a biantennary oligosaccharide, but without the terminal galactoses, should yield a molecular mass of $5068.6 \mathrm{Da}$. The two other components correspond to a glycopeptide with a triantennary carbohydrate, where one of the galactose residues was not removed (calculated molecular mass is $5434.0 \mathrm{Da}$ ) and to an $\mathrm{N}$-terminally truncated version of the triantennary glycopeptide ( $\bullet$ ), when the amino acids Arg and Pro were cleaved (calculated molecular mass is 5018.6 Da). (GN $=\mathrm{N}$-Acetyl-glucosamine; $\mathrm{M}=$ mannose; $\mathrm{G}=$ galactose.)

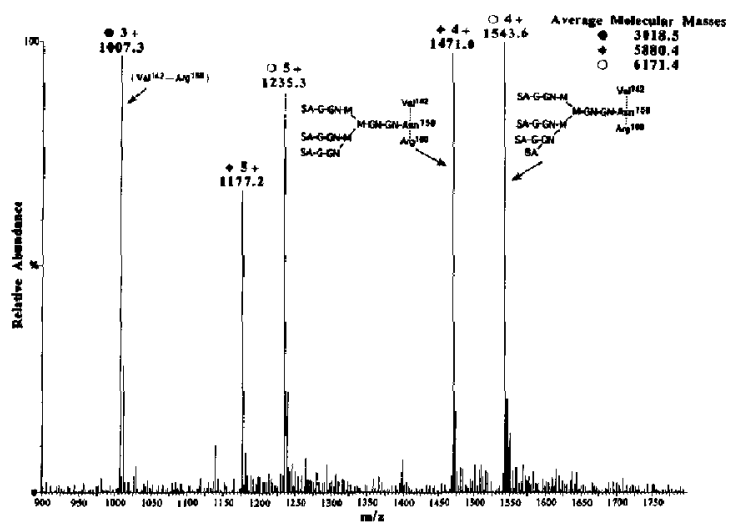

Figure 5. Electrospray mass spectrum of the bovine fetuin tryptic glycopeptides containing Asn ${ }^{158}$. This spectrum was recorded in an LC/ESIMS expcriment from 20 pmoles of the tryptic digest, using water/acetonitrile/TFA as mobile phase for the chromatographic separation. The peptide was observed nonglycosylated, and with complex tri- and tetrasialo triantennary structures at $\mathrm{Asn}^{158}$. The calculated molecular masses for these species are 3017.4, 5880.0, and 6171.3 Da, respectively. (GN $=$ N-Acetylglucosamine; $\mathrm{M}=$ mannose; $\mathrm{G}=$ galactose; $\mathrm{SA}=$ sialic acid.) bearing sialylated complex carbohydrates [11-15] as well as several $O$-linked sites $[11,16-18]$. This glycoprotein was chosen to evaluate our strategy because of the complexity of its glycoform heterogeneity reported previously in studies using primarily NMR techniques employing a variety of strategies in sample preparation [12-15, 19]. In our experience a 20-picomole aliquot of the tryptic digest of bovine fetuin is sufficient to obtain a virtually complete molecular mass map of this glycoprotein, including all $\mathrm{N}$ - and $\mathrm{O}$-linked glycosylation sites, by microbore LC/ESIMS using an acetonitrile gradient, except for the very hydrophilic tripeptide GYK ( $\mathrm{H}-\mathrm{GlyTyrLys-OH}$ ), corresponding to amino acid residues $37-39$. In addition to the expected tryptic peptides, other species were present in this digest mixture. For example, peptides corresponding to sequences 338-341 and 126-141 were identified, indicating that the enzymatic cleavage was not complete (see sequences in Table 1). In addition, a tryptic peptide was detected with a molecular mass of $1460.7 \mathrm{Da}$, and to our surprise, high energy collision-induced dissociation sequence analysis revealed a variant fetuin sequence $316-330$ containing valine at position 324 in place of isoleucine (data not shown). The isoleucinecontaining peptide predicted by the CDNA sequence [11] eluted later and was estimated to be present in a greater amount than the valine variant, suggesting that the peptide containing valine might be derived from a fetuin isoform. In addition, the LC/ESIMS (and SIM) data indicated that the alkylation of cysteine residues at positions 128 and 131 was not complete, since both S- $\beta$-4-pyridylethyl and free cysteinyl analogs were detected, representing sequence 126-141 (Table 1).

In separate LC/ESIMS runs the chromatographic effluent was subjected to collisional activation, thus permitting the detection of those peaks containing glycopeptides from observation of specific lower mass ions [selected ion monitoring (SIM)] characteristic of the presence of generic oligosaccharide moieties. The observation of ions at $m / z 204,366,274$, and 292 indicated the presence of $\mathrm{N}$-acetylhexosamine, hexosyl-N-acetylhexosamine, and sialic acid moieties, respectively, in the SIM data from the LC/ESIMS run (data not shown). Careful comparison of the two chromatograms (LC/SIM and full scan LC/ESIMS) yielded the retention times of glycopeptides. For example, using SIM the first glycopeptide-containing HILC peak detected corresponded to the complete ESIMS spectrum shown in Figure 1. Two Asn ${ }^{138}$-containing glycopeptides eluted in this fraction. Their glycoforms were identified as disialo biantennary, and tri- and tetrasialo triantennary complex carbohydrates, as expecled from NMR data [12, 15]. The next eluting fraction contained peptide 126-141 also bearing the trisialo triantennary carbohydrate structure, but with two free cysteine residues (Table 1).

The complete ESIMS spectrum corresponding to the next $N$-linked fraction identified by SIM is shown in Figure 2. This spectrum provides evidence of the nature of the different oligosaccharides attached to the 
Table 1. Peptides and glycopeptides identified from bovine fetuin tryptic digest using acetonitrile- (A) or alcohol-based (B) solvent systems

\begin{tabular}{|c|c|c|c|c|}
\hline \multicolumn{2}{|c|}{$\begin{array}{l}\text { Retention Time } \\
\text { (min) }\end{array}$} & \multirow[b]{2}{*}{ Sequence [Position] } & \multicolumn{2}{|c|}{ Molecular Mass } \\
\hline A & B & & Calculated & Measured ${ }^{a}$ \\
\hline 19.8 & 8.5 & GSVIOK [214-219] & $630 . \overline{8}$ & 630.7 \\
\hline 20.9 & 19.6 & YFK [338-340] & 456.6 & 456.5 \\
\hline 21.5 & 11.8 & LBPGR [331-335] & 649.8 & 649.7 \\
\hline 21.5 & 6.2 & HLPR $[33-36]$ & 521.6 & 521.6 \\
\hline 21.7 & 16.4 & EVVDPTK [194-200] & 786.9 & 786.8 \\
\hline 23.8 & 18.0 & BDSSPDSAEDVRK [114-126] & 1513.6 & 1513.7 \\
\hline 24.0 & 19.1 & BDSSPDSAEDVR [114-125] & 1385.4 & 1385.6 \\
\hline 24.2 & 18.6 & ALGGEDVR [220-227] & 815.9 & 816.0 \\
\hline 25.4 & 17.9 & AHYDLR [289-294] & 773.9 & 773.9 \\
\hline 26.0 & 19.8 & WWPR [50-531 & 556.7 & 556.6 \\
\hline 26.6 & 20.5 & QYGFBK [208-213] & 850.0 & 850.2 \\
\hline 27.0 & 21.5 & HTLNOIDSVK [40-49] & 1154.3 & 1154.8 \\
\hline 27.2 & 20.7 & BNLLAEK [201-207] & 895.1 & 895.2 \\
\hline 27.9 & 23.6 & $<$ OYGFBK [208-213] & 833.1 & 833.1 \\
\hline 29.1 & 24.0 & QOTOHAVEGDBDIHVLK [86-102] & 2026.3 & 2026.3 \\
\hline 29.6 & 25.1 & < QOTQHAVEGDBDIHVLK [86-102] & 2009.3 & 2009.3 \\
\hline 29.6 & 26.3 & TPIVGOPSVPGGPVR $[316-330]^{\mathrm{b}}$ & 1460.7 & 1460.6 \\
\hline 30.5 & 27.4 & TPIVGQPSIPGGPVR [316-330] & 1474.7 & 1474.8 \\
\hline 30.9 & 26.9 & IPLDPVAGYK $[1-10]$ & 1072.3 & 1072.4 \\
\hline 31.0 & 25.9 & YFKI [338-341] & 569.7 & 569.7 \\
\hline \multirow[t]{2}{*}{31.5} & 28.3 & KLBPDBPLLAPLNDSR [126-141] & & \\
\hline & 31.1 & $\begin{array}{l}\text { Hex }_{6} \mathrm{HexNAc}_{5} \mathrm{SA}_{3} \\
\text { LBPDBPLLAPLNDSR }[127-141]\end{array}$ & 4828.0 & 4828.0 \\
\hline 31.7 & 28.8 & $\begin{array}{l}+\mathrm{Hex}{ }_{6} \mathrm{HexNAc}_{5} \mathrm{SA}_{4} \\
\text { LBPDBPLLAPLNDSF [1 } 27-141]\end{array}$ & 4991.1 & 4991.6 \\
\hline 31.8 & 28.3 & $\begin{array}{l}+\mathrm{Hex}_{6} \mathrm{HexNAc}_{5} \mathrm{SA}_{3} \\
\text { LBPDBPLLAPLNDSR [1 } 27-141]\end{array}$ & 4699.8 & 4700.5 \\
\hline 31.8 & 27.4 & $\begin{array}{l}+\mathrm{Hex}_{5} \mathrm{HeXNAC}_{4} \mathrm{SA}_{2} \\
\text { HTFSGVASVESSSGEAF- }\end{array}$ & 4043.2 & 4043.5 \\
\hline 31.9 & & HVGK $[295-315]$ & 2120.3 & 2120.6 \\
\hline 33.4 & 30.8 & QDGQFSVLFTK [103-113] & 1269.4 & 1269.6 \\
\hline \multirow[t]{2}{*}{33.5} & 32.4 & KLCPDCPLLAPLNDSR [126 -141] & & \\
\hline & 33.0 & $\begin{array}{l}+\mathrm{Hex}_{6} \mathrm{HexNAc}_{5} \mathrm{SA}_{3} \\
\text { EPABDDPDTEQAALAAV- }\end{array}$ & 4617.7 & 4616.4 \\
\hline 34.4 & & DYINK $[11-32]$ & 2454.7 & 2455.0 \\
\hline 34.5 & 34.2 & $<$ QDGOFSVLFTK $[103-113]$ & 1252.4 & 1252.2 \\
\hline \multirow[t]{2}{*}{36.2} & 34.7 & IPLDPVAGYKEPABDDP- & & \\
\hline & & DTEOAALAAVDYINK [ 1 -32] & 3508.9 & 3509.7 \\
\hline \multirow[t]{2}{*}{37.3} & 46.5 & $\begin{array}{l}\text { VTBTLFOTOPVIPOPOPDGAEAEAPSAVPDA- } \\
\text { AGPTPSAAGPPVASW } N G P S V \text { VAVPLLHR }\end{array}$ & & \\
\hline & & $\mid$ Iex ${ }_{3} \mid$ lexNAc $S_{3} \mathrm{SA}_{4}[228-288]$ & 8327.1 & 8328.7 \\
\hline 37.4 & 38.7 & VTETLFOTOPVIPOPQPDGAEAEAPSAVPDA- & & \\
\hline 37.5 & 38.8 & $\begin{array}{l}\text { AGPTPSAAGPPVASVVGPSVVAVPPLHR } \\
\text { +Hex }{ }_{3} \mathrm{HexNAC}_{3} \mathrm{SA}_{3}[228-288] \\
\text { VTBTLFOTQPVIPQPQPDGAEAEAPSAVPDA- } \\
\text { AGPTPSAAGPPVASVVVGPSVVAVPLPLHR }\end{array}$ & 8035.8 & 8036.1 \\
\hline 37.7 & 38.2 & $\begin{array}{l}+\mathrm{Hex}_{2} \mathrm{HexNAG}_{2} \mathrm{SA}_{3}[228-288] \\
\text { VTETLFOTOPVIPQPOPDGAEAEAPSAVPDA- } \\
\text { AGPTPSAAGPPVASWWGPSWVAVPLPLHR }\end{array}$ & 7670.4 & 7670.7 \\
\hline \multirow[t]{2}{*}{38.5} & 38.4 & $\begin{array}{l}+\mathrm{Hex}_{2} \mathrm{HexNAc}_{2} \mathrm{SA}_{2} \text { [228-288] } \\
\text { RPTGEVYDIEIDTLETIBHVLDPTPLANBSVR }\end{array}$ & 7379.2 & 7380.1 \\
\hline & 37.8 & $\begin{array}{l}+ \text { Hex }_{6} \mathrm{HexNAc}_{5} \mathrm{SA}_{4}[54-85] \\
\text { RPTGEVYDIEIDTLETTBHVLDPTPLANBSVR }\end{array}$ & 6923.3 & 6924.4 \\
\hline 38.6 & $3 \% .2$ & $\begin{array}{l}+ \text { Hex }_{6} \mathrm{HeXNAC}_{5} \mathrm{SA}_{3}[54-85] \\
\text { RPTGEVYDIEID TLE I I BHVLUPTPLANBSVR }\end{array}$ & 6631.9 & 6632.4 \\
\hline 38.6 & & $+\mathrm{Hex}_{6} \mathrm{HexNAC}_{5} \mathrm{SA}_{2}[54-85]$ & 6340.5 & 6642.4 \\
\hline \multirow[t]{2}{*}{38.6} & 37.2 & RPTGEVYDIEIDTLETTBHVLDPTPLANBSVR & & \\
\hline & & $+\mathrm{Hex}_{6} \mathrm{HexNAc}_{4} \mathrm{SA}_{2}[54-85]$ & 5975.3 & 5976.0 \\
\hline 40.7 & $\sim 41$ & $\begin{array}{l}\text { VHAVEVALATFNAESNGSYLQLVEISR } \\
+ \text { Hex }_{6} \mathrm{HexNAC}_{5} \mathrm{SA}_{4}[142-169]\end{array}$ & 6171.3 & $\begin{array}{l}6172.1 \\
(\text { continued })\end{array}$ \\
\hline
\end{tabular}


Table 1. (continued)

\begin{tabular}{|c|c|c|c|c|}
\hline \multicolumn{2}{|c|}{$\begin{array}{c}\text { Retention Time } \\
\text { (min) }\end{array}$} & \multirow[b]{2}{*}{ Sequence [Position] } & \multicolumn{2}{|c|}{ Molecular Mass } \\
\hline A & B & & Calculated & Measured ${ }^{a}$ \\
\hline $\begin{array}{l}40.7 \\
41.3 \\
45.0\end{array}$ & $\begin{array}{l}\sim 41 \\
39.5 \\
41.1\end{array}$ & $\begin{array}{l}\text { VVHAVEVALATFNAESNGSYLQLVEISR } \\
\text { +Hex }_{6} \text { HeXNAC }_{6} \text { SA }_{3}[142-169] \\
\text { WHAVEVALATFNAESNGSYLQLVEISR } \\
\text { [142-169] } \\
\text { AQFVPLPVSVSVEFAVA- } \\
\text { ATDBIAK }[170-193]\end{array}$ & $\begin{array}{l}5880.0 \\
3017.4 \\
2568.1\end{array}$ & $\begin{array}{l}5880.8 \\
3017.7 \\
2568.4\end{array}$ \\
\hline $\begin{array}{l}\mathrm{B}=\mathrm{S}-\beta-4 \\
<\mathrm{Q}=\mathrm{PY} \\
\mathrm{a} \text { These } \mathrm{m} \\
\text { Da). } \\
\text { Sites for } \\
\text { Retention } \\
\text { Cetonitrile } \\
\text { cetdition of }\end{array}$ & de & $\begin{array}{l}\text { ned from a single LC /ESIMS experiment } \\
\text { analysis. } \\
\text { rmined from these data. } \\
\text { m the ion extraction chromatograms } \\
\text { an approximate twa min delay between } \\
\text { umn split reguired. For the alcohol-based }\end{array}$ & $\begin{array}{l}\text { teristic ion } \\
\text { lass spectro } \\
\text { e this delay }\end{array}$ & $\begin{array}{l}\text { tobile phase 'Figure } \\
\text { component. In the } \\
\text { ction caused by the } \\
0.4 \text { min. }\end{array}$ \\
\hline
\end{tabular}

peptide sequence 54-85. Multiply protonated ion series are present at $m / z \quad 997.0$ and 1196.1; 1057.8 and $1269.3 ; 948.7,1106.5,1327.4$, and 1659.4; 1154.7 and 1385.7 , representing disialo biantennary as well as di-, tri-, and tetrasialo triantennary oligosaccharides at $\mathrm{Asn}^{81}$, respectively. The corresponding molecular weights determined for these glycopeptide glycoforms were 5975.0, 6341.0, 6632.9, and 6922.9 Da.

In order to establish that these masses in fact correspond to molecular masses of the glycoforms postulated above and are not fragments of one another, the glycopeptide HPLC fractions were collected preparatively from 1 nmole of the tryptic digest and subjected to sequential exoglycosidase digestions followed by LC/ESIMS analysis at each stage. These experiments were carried out for the fraction discussed above since the masses shown in Figure 2 indicated the presence of differentially sialylated triantennary structures, as well as a previously undescribed (at this glycosylation site) disialo biantennary structure on the 54-85 sequence.

First this fraction (Figure 2) was digested with neuraminidase from Arthrobacter ureafaciens in order to remove any terminal sialic acid residues. Figure 3 shows the results of this digestion revealing two components with molecular masses 5392.3 and $5757.5 \mathrm{Da}$, corresponding to the same amino acid sequences bearing putative asialo biantennary and asialo triantennary complex carbohydrate structures. In order to define these structures, these truncated glycopeptides were digested with $\beta$-galactosidase from Streptococcus pneumoniae, $\beta$-N-acetylhexosaminidase (from chicken liver), and $\alpha$-mannusidase (from Jack bean) [20]. The results from the second digestion step are shown in Figure 4. For this digestion, the $\beta$-galactosidase used was expected to cleave only Gal $\beta(1,4)$ GlcNAc(GalNAc) linkages. However, ions were detected yielding molecular masses 5434.7, 5272.3, 5068.9, and 5019.1 Da. The highest value observed $(5434.7 \mathrm{Da})$ indicates that one of the galactose units has not been removed from the triantennary structure. This observation is consistent with the linkage specificity of the enzyme, since the tet- rasialo structure contains $\mathrm{Gal} \beta(1,3)$ GlcNAc linkages [15] and thus, some incomplete cleavage should be expected. However, repeating this experiment with a longer incubation time and another aliquot of the same exoglycosidase resulted in the removal of all galactose residues from these glycopeptides, indicating that in fact this enzyme cleaved both linkages but with a different reaction rate (data not shown). The component at mass $5272.3 \mathrm{Da}$ corresponds to amino acid sequence $54-85+\mathrm{Hex}_{3} \mathrm{HexNAc}_{5}$, indicating that three galactose residues have been removed from the triantennary carbohydrate structure, as expected. Mass 5068.9 Da corresponds to the glycopeptide with a biantennary oligosaccharide, again with two galactose residues removed, as expected. The glycopeptide with molecular mass 5019.1 Da indicates that this $\beta$-galactosidase enzyme preparation exhibited some peptidase activity, since this species is the N-terminally truncated version of the major component, with the $\mathrm{N}$ terminal amino acid residues, Arg and Pro, removed. Two consecutive, additional digestion steps-using enzymes specific for GIcNAc $\beta(1-3,4) R$, and Man $\alpha(1-2,3,6)$ Man-yielded a species with one mannose residue attached to the chitobiose core, and thus established the presence of both complex biantennary and trianterniary carbohydrates at $\mathrm{Asn}^{\mathrm{B1}}$ (data not shown).

Similarly, another glycopeptide containing peak was identified by SIM and the corresponding full scan ESIMS spectrum is shown in Figure 5. This spectrum contains masses attributed to both the unglycosylated tryptic peptide 142-169 ( $\mathrm{m} / \mathrm{z} 755.5$ and 1007.3, MW $=3018.5 \mathrm{Da}$ ), and those chromatographically unresolved glycoforms bearing a trisialo and tetrasialo triantennary complex carbohydrate structure (ions at $m / z 1177.2$ and 1471.0, MW $=5880.4 \mathrm{Da}$; ions at $m / z 1235.3,1543.6, \mathrm{MW}=6171.4 \mathrm{Da}$, respectively). The tetrasialylated glycopeptide comprises at least $50 \%$ of the glycopeptide mixture in this fraction as evidenced by relative peak height. Earlier glycopeptide studies carried out in this laboratory have demon- 
strated that there was good agreement between the relative ion intensities representing the different glycoforms and their relative distribution [4].

As is well known, using the acetonitrile-based mobile phase glycopeptide separation appears to be dominated by the properties of the peptide chain, and therefore glycopeptide glycoforms are usually not separated. Hence, we have explored the use of an alternative mobile phase containing an aqueous mixture of small organic alcohols and formic acid (water/ ethanol/propanol/formic acid) for separation of the glycoprotein digest. The UV absorbance chromatograms observed for both mobile phases are shown in Figure 6. The upper panel illustrates the separation using the acetonitrile-based solvent system. The lower panel shows the altered elution profile of the components using the water/ethanol/propanol/formic acid mobile phase. The retention times for the components in the two different solvent systems were determined by ESIMS detection and are presented in Table 1. For example, glycoforms of glycopeptide 127-141 coeluted in the water/acetonitrile system (Figure 6a). However, these glycoforms are clearly separated in the alcoholbased mobile phase. Thus, the glycopeptide species bearing a disialo biantennary oligosaccharide eluted first (at $\sim 27.9 \mathrm{~min}$, Figure $6 \mathrm{~b}$ ), followed by the glycopeptide with the trisialo triantennary structure ( $\sim$ $28.4 \mathrm{~min}$, Figure 6b), and finally the tetrasialylated species at $\sim 30.7 \mathrm{~min}$ (Figure $6 \mathrm{~b}$ ). Similar separation of the different glycoforms was observed for the glycopeptide series containing $\mathrm{Asn}^{81}$ as well (Figure $6 \mathrm{~b}$ ). The glycopeptide species 54-85 with the disialo biantennary carbohydrate structure eluted first, and it was also separated from the major glycoform with the trisialo triantennary carbohydrate structure (Figure 7). Finally, glycopeptides corresponding to sequence

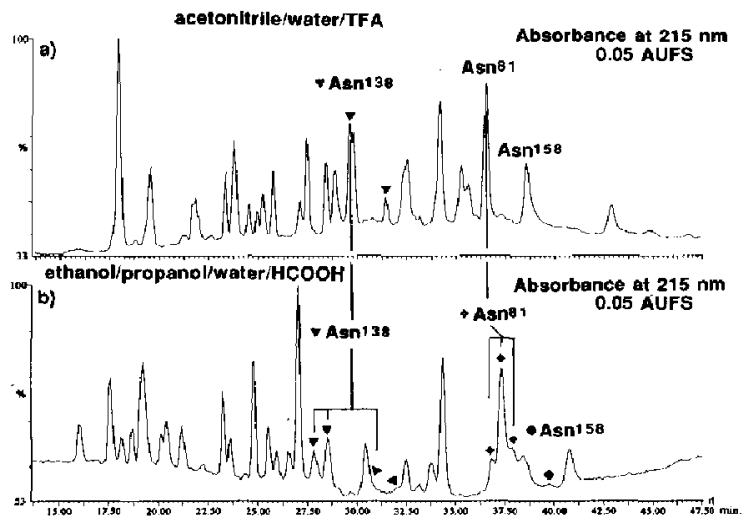

Figure 6. HI'LC chromatograms of the tryptic digest of bovine fetuin comparing the two solvent systems. Panel (a) shows the UV absorbance chromatogram for the separation of 20 pmoles of the fetuin tryptic digest in water/acetonitrile/TFA mobile phase. Panel (b) shows the UV absorbance chromatogram of another aliquot of the digest separated using the ethanol/propanol/ water/formic acid system. Peaks corresponding to glycopeptides are labeled.

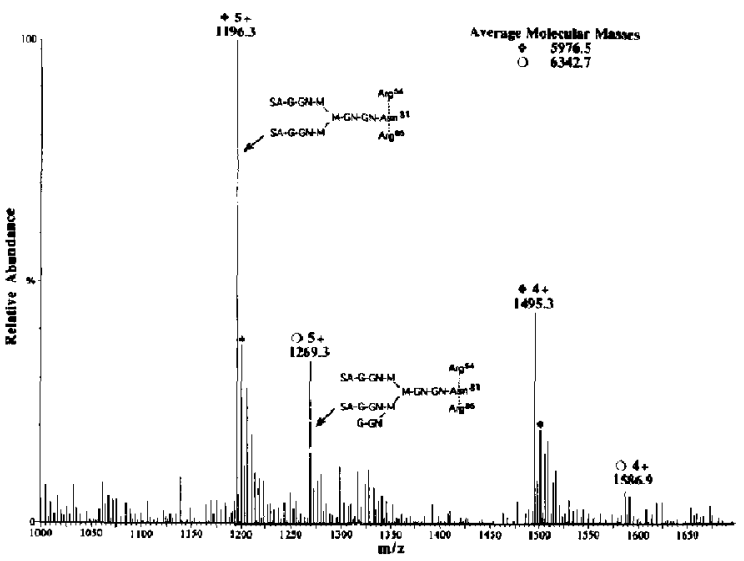

Figure 7. Electrospray mass spectrum of the first eluting glycoforms of glycopeptide 54-85 in the alternative solvent system. The calculated molecular masses are 5975.3 and $6340.5 \mathrm{Da}$, respectively. For the major component multiply charged sodiumadduct peaks were detected (labeled with asterisks). The presence of metal-adduct peaks can aid the charge-state determination [23], (GN $=\mathrm{N}$-Acetyl-glucosamine; $\mathrm{M}=$ mannose; $\mathrm{G}=$ galactose; $\mathrm{SA}=$ sialic acid.)

142-169 were not as clearly resolved, but from the mass chromatograms (not shown) the unglycosylated peptide eluted first, followed by the tri- and tetrasialylated species. The recovery of these glycopeptides $\left(A s n^{158}\right)$ was very poor in this mobile phase, the glycoforms of this hydrophobic sequence eluted as wide peaks, and the tetrasialylated species was frequently not observed.

The LC/ESIMS experiments were carried out using two different electrospray mass spectrometers, as reported in Instrumentation. Our data show that a definite shift towards lower charge states, that is, higher masses, was observed in both mobile phases when the data were obtained on the Platform mass spectrometer. For example, the glycopeptide ${ }^{54} \mathrm{Arg}-\mathrm{Arg}^{85}$ bearing a disialo bianteruary oligosaccharide was represented by ions at $n / z 977.0(6+)$ and $1196.1(5+)$ when detected on the Bio-Q mass spectrometer (Figure 2), while ions at $m / z 1196.3(5+)$ and $1495.3(4+)$ (Figure 7) were detected when analyzed by the other instrument.

\section{Discussion}

Bovine fetuin is a well-characterized glycoprotein consisting of 341 amino acids [11]. Partial mass mapping (using fast-atom bombardment) of its tryptic digest has been reported [21]. It contains three $N$-linked glycosylation sites whose carbohydrate structures and site heterogeneity has been studied extensively by NMR [12-15]. Similarly, sites for $\mathcal{O}$-glycosylation and attached oligosaccharides have been described [16-18]. NMR studies on the free $N$-linked carbohydrates of bovine fetuin revealed that the tetra- or pentasialo oligosaccharides were related in having a $\mathrm{Gal} \beta(1,3)$ 
(NeuAc $\alpha 2,6$ )GlcNAc $\beta(1,4)$ Man moiety [15]. Further, NMR studies of fetuin asialo glycopeptides showed that approximately $20 \%$ of the oligosaccharides linked to $A s n^{81}$ exhibited this branch. For glycosylation sites $\mathrm{Asn}^{138}$ and $\mathrm{Asn}^{158}$ the proportions of this structure observed were $40 \%$ and $60 \%$, respectively [12]. Based on these results the highest percentage of penta- and tetrasialo triantennary oligosaccharides can be expected for $\mathrm{Asn}^{158}$, followed by $\mathrm{Asn}^{138}$, while glycopeptides containing $\mathrm{Asn}^{81}$ should exhibit mostly trisialo triantennary carbohydrates.

The most comprehensive LC/ESIMS study on bovine fetuin glycopeptides has been carried out by S. A. Carr et al. [3]. In this work fetuin (from Sigma) and asialo fetuin were studied by digestion with a mixture of trypsin and endoprotease Asp-N. This enzyme combination yields glycopeptides significantly smaller than those generated by solely using trypsin. Thus, the masses occur in regions of the mass scale where sensitivity of quadrupole instruments is optimal because the absolute sensitivity of quadrupole filters decreases with increasing mass, especially above 1000 Da. Glycopeptides containing trisialo triantennary complex carbohydrates were observed for $\mathrm{Asn}^{81}$ and $\mathrm{Asn}^{138}$. However, only asialo glycopeptides were detected for glycopeptides containing $\mathrm{Asn}^{158}$, and the penta- and tetrasialo structures expected based on the NMR studies were not observed for any of the glycosylation sites. It is interesting that disialo biantennary structures were detected for both $\mathrm{Asn}^{81}$ and $\mathrm{Asn}^{138}$, since a disialo-biantennary structure has not been described previously at $\mathrm{Asn}^{81}$. It was noted in this work that additional studies would be necessary to establish this novel structure at $\mathrm{Asn}^{81}$ because this species was detected during "orifice stepping" used to induce carbohydrate fragmentation, thus observation of a smaller mass signal could have been due to fragmentation processes [3].

In the present study using the water/acetonitrile/ TFA mobile phase, all the major sialylated tryptic glycopeptides were detected. The relative amounts of tetra- and trisialo compounds recorded in these experiments were consistent with the results reported based upon NMR data $[12,15]$. For example, among the $\mathrm{Asn}^{158}$ glycopeptides, the tetrasialylated species was detected as the major component (Figure 5). In addition, this particular sequence was detected unglycosylated as well, implying that glycopeptides representing this amino acid region are present at lower concentrations than their counterparts for $\mathrm{Asn}^{81}$ and $\mathrm{Asn}^{138}$, which were found to be completely glycosylated in our studies. This may explain why no sialylated glycopeptides were observed by Carr et al. for Asn ${ }^{158}$. Pentasialo carbohydrate structures were not observed in our experiments. Based upon studies of the $\mathrm{N}$-linked carbohydrates of commercially available fetuin samples using high $\mathrm{pH}$ anion exchange chromatography the pentasialo triantennary oligosaccharides represent only $\sim 1 \%$ of the total $N$-linked structures in Gibco preparations [22]. This level of pentasialo glycopeptides would most likely be below our detection limit.

Sequential digestion by residue and linkage specific exoglycosidases followed by LC/ESIMS analysis offers a sensitive tool for more complete small scale glycopeptide glycoform characterization. Using this method, the number of different glycoforms for each glycopeptide can be identified, the order and identity of the specific carbohydrate units comprising the oligosaccharides can be determined, and in some cases the position and stereochemistry of their linkage can be determined as well. The existence of a disialo biantennary oligosaccharide at $\mathrm{Asn}^{81}$ in bovine fetuin was established clearly in this manner. Additional proof was provided by LC/ESIMS analysis of the same tryptic digest using a different solvent system, which provided separation of some glycoforms, demonstrating independently that this lower molecular weight glycopeptide was indeed a real component of the mixture and not a product of induced fragmentation. We have observed that the water/propanol/ethanol/formic acid solvent system significantly altered the elution order of the peptides and glycopeptides. In fact, to our surprise we found that the glycoforms of the glycopeptides eluted in reverse order and were chromatographically resolved. Experiments to ascertain whether lowering the hydrophobicity of glycopeptide 142-169 by enzymatic truncation would shorten its retention time and improve the glycoform separation are in progress.

In summary, the ethanol/propanol/water/formic acid mobile phase offers a simple alternative for LC/ESIMS analyses because of its compatibility with both the chromatographic separation of peptides and electrospray ionization. In addition, no other reversedphase HPLC methods have been reported for separation of glycopeptide glycoforms. The reasons for the reverse elution order and separation of glycoforms in this solvent system are not yet understood. Nevertheless, this method may become a valuable tool for unraveling the complexity of glycoform heterogeneity. Similarly, this mobile phase can be used for the analysis of complicated protein digests. As demonstrated, this alternative solvent system may yield a better separation for components which coelute in the water/ acetonitrile/TFA solvent system. This better chromatographic resolution can lead to improved mass spectrometric detection sensitivity for components which are "handicapped," either because of their size (glycopeptides, peptides of large molecular weights, e.g., $>4000 \mathrm{Da}$ ) or because they are present in less than stoichiometric quantities such as posttranslationally or xenobiotically modified peptides.

\section{Acknowledgments}

The authors would like to thank $R . R$. Townsend for supplying the fetuin sample and valuable discussions. We are indebted to Fisons/VG for loan of the Platform electrospray mass spectrome- 
ter. This work was supported by grants from the National Institutes of Health, National Center for Research Resources (grant no. RR01614) and National Institute of Diabetes and Digestive and Kidney Diseases (grant no. AM 27643) and National Institute of Environmental Health Sciences (grant no. ES04705, to A. L. B.), and National Institutes of Health Training (grant no. NSO7219, to S. C. H.).

\section{References}

1. Ling, V. L.; Guzzetta, A. W.; Canova-Davis, E.; Stults, J. T.; Hancock, W. S. Anal. Chem. 1991, 63, 2909-2915.

2. Thibault, P.; Watson, D. C.; Young, N. M. In Proceedings of the 41st ASMS Conference on Mass Spectrometry and Allied Topics, San Francisco, CA. 1993, p. 613.

3. Carr, S. A.; Huddleston, M. J.; Bean, M. F. Protein Science 1993, 2, 183.

4. Medzihradszky, K. F.; Settineri, C. A.; Maltby, D. A.; Burlingame, A. L. In Techniques in Protein Chemistry IV; Angeletti, R. H., Ed. Academic Press: San Diego, 1993; pp. 117-125.

5. Huddleston, M. J.; Bean, M. F.; Carr, S. A. Anal. Chem. 1993, $65,877-884$.

6. Carr, S. A.; Barr, J. R.; Roberts, G. D.; Anumula, K. R.; Taylor, P. B. In Methods in Enzymology, Vol, 193; McCloskey, J. A., Ed. Academic Press: San Diego, 1990; pp. 501-518.

7. Medzihradszky, K. F.; Schindler, P. A.; Ding, A. R.; Maltby, D. A.; Burlingame, A. L. In Praceedings of the Kyoto ' 92 International Conference an Biological Mass Spectrometry, Matsuo, T., Ed. San-ei Publishing Co.: Kyoto, 1992; pp. 42-43.

8. Feldhoff, R. In Techniques In Protein Chemistry I; Villafranca, J. J., Ed. Academic Press: San Diego, 1991; pp. 55-63.

9. Stacey, C. C.; Sheehan, E.; Mitrovitch, S. Electrospray LC / MS
Characterization of Peptides, Poster Presentation, Seventh Symposium of the Protein Society, San Diego, July 24-28, 1993.

10. Griffin, P. R.; Colfman, J. A.; Hood, L. E.; Yales, J. R, Iml. I. Mass Spect. and Ion Processes 1991, 111, 131-149.

11. Dziegielewska, K. M.; Brown, W. M.; Casey, S. J.; Christie, D. L.; Foreman, R. C.; Hill, R. M.; Saunders, N. R. J. Biol. Chem. 1990, 265, 4354-4357.

12. Townsend, R. R.; Hardy, M. R.; Wong, T. C.; Lee, Y. C. Biochemistry 1986, 25, 5716-5725.

13. Townsend, R. R; Alai, M.; Hardy, M. R; Fenselau, C. C. Anal. Biochem. 1988, 171, 180-191.

14. Bendiak, B.; Harris-Brandts, M.; Michuik, S. W.; Carver, J. P.; Cumming, D. A. Biochemistry 1989, 28,64916499.

15. Cumming, D. A.; Hellerqvist, C. G.; Harris-Brandts, M.; Michnick, S. W.; Carver, J. P.; Bendiak, B. Biochemistry 1989, 28, 6500-6512.

16. Spiro, R. G.; Bhoyhroo, V. D. J. Biol. Chem. 1974, 249, 5704-5717.

17. Edge, A. S. B; Spiro, R. G. J. Biol. Chem. 1987, 262, 16, 135-16,141.

18. Townsend, R. R.; Medzihradszky, K. F.; Hardy, M. R.; Rohrer, J.; Burlingame, A. L. XI International Symposium on Glycoconjugates, Poster Presentation, Toronto, 1991.

19. Townsend, R. R. In Biological Mass Spectrometry; Burlingame, A. L.; McCloskey, J. A., Eds. Elsevier: Amsterdam, Netherlands, 1990, Pp, 375-395.

20. Settineri, C. A.; Burlingame, A. L. In Techniques In Protein Chemistry V; Crabb, J. W, Ed. Academic Press: San Diego, 1994; pp. 97-104.

21. Smith, K. D.; Harbin, A. M.; Carruthers, R. A.; Lawson, A. M.; Hounsell, E. F. Biomed. Chrom. 1990, 4, 261-266.

22. Townsend, R. R.; Hardy, M. R.; Cumming, D. A.; Carver, J. P.; Bendiak, B. Anal. Biochem. 1989, 182, 1-8.

23. Senko, M. W.; Beu, S. C.; McLafferty, F. W. I. Am. Soc. Mass Spectrom. 1993, 4, 828-830. 